\title{
氯化两面针碱合成方法的改进
}

\author{
许旭升 ${ }^{a, b}$ 刘志千 ${ }^{b}$ 郡文浩 ${ }^{b}$ 叶 雯 ${ }^{b}$ \\ 孙青壟 $b$ 何薇微 $*, a$ 张卫东*,, ,,$b$ \\ ( ${ }^{a}$ 华东理工大学药学院 上海 200237) \\ ( 第二军医大学药学院 上海 200433)
}

\begin{abstract}
摘要 以 6-溴-3,4-二甲氧基苯甲醛和 6,7-亚甲二氧基-1-萘胺为起始原料, 经还原胺化、分子内 Heck 偶联、氧化芳构化、 甲基化以及离子交换等五步反应，完成了氯化两面针碱的合成，并对关键步骤分子内 Heck 偶联反应条件进行了探索， 结果表明该偶联反应在醋酸钯、三(邻甲苯基)膦和 $N, N^{\prime}$-二甲基甲酰胺体系中反应效果最佳, 收率显著提高. 该优化条 件为结构类似苯并 $[c]$ 菲啶类生物碱母核结构的构建提供了借鉴.
\end{abstract}

关键词 氯化两面针碱; 分子内 Heck 偶联反应; 合成改进

\section{Process Improvement on the Synthesis of Nitidine Chloride}

\author{
Xu, Xusheng ${ }^{a, b} \quad$ Liu, Zhiqian $^{b} \quad{\text { Shao, } \text { Wenhao }^{b} \quad \text { Ye, } \mathrm{Ji}^{b} \quad \text { Sun, Qingyan }}^{b}$ \\ He, Weiwei* ${ }^{*, a} \quad$ Zhang, Weidong*,a,b \\ ( ${ }^{a}$ College of Pharmacy, East China University of Science and Technology, Shanghai 200237) \\ ( ${ }^{b}$ College of Pharmacy, The Second Military Medical University, Shanghai 200433)
}

\begin{abstract}
Nitidine chloride was synthesized via reductive amination, intramolecular Heck coupling reaction, aromatization, methlyation and ion exchange, using 6-bromine-3,4-dimethoxybenzaldehyde and 6,7-methylenedioy-1-naphthylamine as starting material. The key intramolecular Heck coupling reaction was optimized, and acetic acid palladium(II) $\left[\mathrm{Pd}(\mathrm{OAc})_{2}\right]$, tris(2-methylphenyl)phosphine $\left[\mathrm{P}(o \text {-tol })_{3}\right]$ and $N, N$-dimethylformamide (DMF) system was confirmed as the most efficient conditions. The total yield was increased significantly, and the optimal conditions may also be applied to the synthesis of other benzo[c]phenanthridine alkaloid.
\end{abstract}

Keywords nitidine chloride; intramolecular Heck reaction; synthesis

氯化两面针碱(nitidine chloride, 1 , 图 1)是从芸香科 花椒属植物两面针的干燥根中分离得到的苯并 $[c]$ 菲啶 类生物碱, 具有抗肿瘤、抗炎等药理作用 ${ }^{[1,2]}$. 研究显示 氯化两面针碱的抗肿瘤作用机制包括抑制拓扑异构酶 I 和 II、诱导细胞调亡、阻滞细胞周期和抑制 DNA 连接 酶的活性 ${ }^{[3]}$ 等, 其作为抗肿瘤化合物研究开发受到许多 药物化学家的重视.<smiles></smiles>

图 1 氯化两面针碱(1)

Figure 1 Nitidine chloride (1)
为克服氯化两面针碱提取分离收率低的缺陷，化学 家致力于发展其全合成方法 ${ }^{[4]}$, 其中 Cushman 的团队在 1978 年报道了一条开创性的合成路线 ${ }^{[5]}$, 他们以 6,7 二 二 甲氧基-3,4-二氢-2-苯并吡喃-1,3-二酮和 3,4-亚甲二氧基 苯亚甲基甲胺通过缩合环化、羧基构型翻转、ArndtEister 反应和分子内 Friedel-Crafts 酰化反应等合成氯化 两面针碱, 步骤较短且产率较高, 但是合成过程中使用 剧毒试剂叠氮甲烷，增加了反应操作的风险性。而 Suzuki 等报道的全合成路线 ${ }^{[6,7]}$ 最为经典, 该路线起始 原料相对易得，反应条件温和且操作简便，但其关键的 环化偶联反应收率仅为 $17 \%$, 导致路线总得率极低. Lautens 等 ${ }^{[8]}$ 探索了一条富有创新性的合成方法学, 他们

\footnotetext{
*E-mail: heweiwei@ecust.edu.cn

Received December 20, 2014; revised January 28, 2015; published online February 10, 2015.

Project supported by the NSFC (No. 81230090), the Shanghai Leading Academic Discipline Project (No. B906).

国家自然科学基金(81230090)、上海市重点学科建设(No. B906)资助项目.
} 
以 6,7-亚甲二氧基-1-三氟甲磺酸萘酯和(2-溴-4,5-二甲 氧基苯亚甲基)-三甲基硅烷基酰胺为环合的起始原料, 在醋酸钯的催化下一步环合生成两面针碱, 但是该合成 方法的两个原料都需要合成, 反应步骤多, 条件苛刻, 使得总体产率下降. 所以本文选择在经典的 Suzuki 合成 路线优化, 提高其关键环化反应的产率, 从而改善总体 反应的收率. Majumdar 等 ${ }^{[9]}$ 近期报道用钯试剂催化二芳 基偶联合成芳香杂环化合物. 这为本文提供合成氯化两 面针碱的新思路. 在 Suzuki 的合成路线基础上, 本文引 入了分子内的 Heck 偶联反应替代原有的环化反应, 有 效地提高了目标化合物的产率(Scheme 1).

\section{1 结果与讨论}

\section{1 关键中间体 4 的合成}

6-溴-3,4-二甲氧基苯甲醛(2)是商业可得的化合物, 6,7-亚甲二氧基-1-菜胺(3) 可以通过对 6,7-二羟基-1-硝基
萗还原胺化 ${ }^{[10]}$ 和邻二羟基亚甲基化很方便地合成. 本 文以醛基化合物 2 和氨基化合物 $\mathbf{3}$ 为起始原料，将两个 底物溶于甲醇中，并用乙酸将反应溶剂调到弱酸性，在 室温下两个底物缩合生成席夫碱化合物, 直接在原有体 系中加入还原剂氰基氢化钠 $\left(\mathrm{NaBH}_{3} \mathrm{CN}\right)$, 还原得到关键 中间体化合物 $N$-[(2-溴-4,5-二甲氧基苄基 $)]-$ 苯并 [2,3- $d]-1,3$-二恶茂-5-胺(4). 本文以 “一锅法” 合成关键 中间体，使得操作更加简单，反应产率更高.

\subsection{Heck 偶联反应的条件优化}

得到关键中间体后，开始探索分子内的 Heck 偶联 反应条件. 本文对钯催化剂、有机磷配体、反应溶剂和 反应时间做了系统的篮选，结果如表 1 所示. 发现增大 反应溶剂的极性可以明显改善反应的效果，在非极性溶 剂苯和二甲苯, Heck 偶联反应的效果非常差, 但在极性 溶剂乙腈和 $N, N^{\prime}$-二甲基甲酰胺反应效果明显改善, $N, N^{\prime}$-二甲基甲酰胺为最佳的反应溶剂. 从表 1 中 Entries<smiles>COc1cc2c(cc1CNc1ccc(CNc3cccc4cc5c(cc34)OCO5)cc1OC)OCO2</smiles>

(1) Toluene, $\triangle$

(2) $\mathrm{Me}_{2} \mathrm{NHBH}_{3}, \mathrm{AcOH}$ $76 \%$

(1) warm up

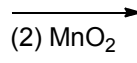
$17 \%$<smiles>COc1cc(CNc2cccc3cc4c(cc23)OCO4)cc(Br)c1OC</smiles><smiles>COc1cc2cnc3c4cc5c(cc4ccc3c2cc1OC)OCO5</smiles>

\section{(1) methyl-2-nitrobenzene
sulfonate}

(2) deprotection $55 \%$<smiles></smiles>

图式 1 Suzuki 的氯化两面针碱合成路线

Scheme 1 Total synthesis of nitidine chloride by Suzuki<smiles>CCCCC1Oc2cc(Br)c(Br)cc2O1</smiles>

\section{$\overrightarrow{1,2-\text { dicholoethane, r.t. }}$} $85 \%$

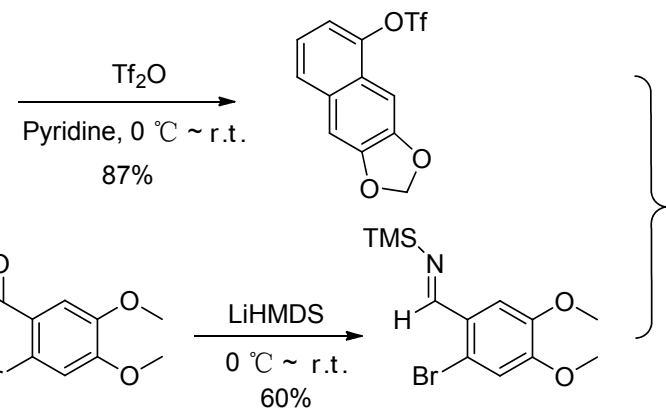

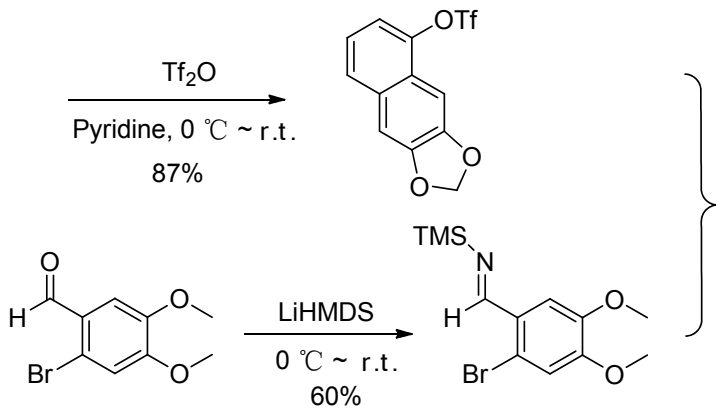

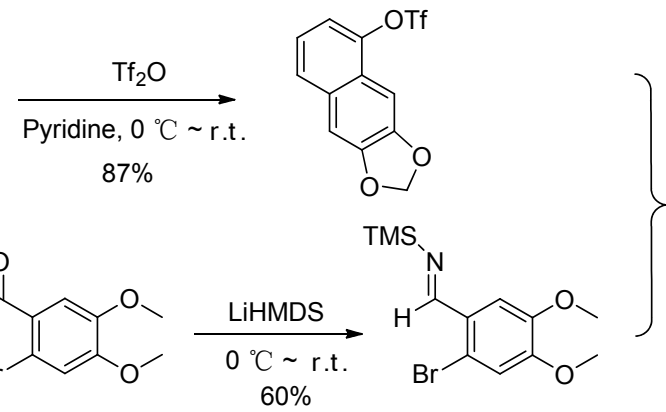
TMS<smiles>CCCCC</smiles><smiles>COc1cc2c(OC)cc3c(c2cc1OC)=CC=c1ccc2cc4c(cc2c1=NC=3)OCO4</smiles>

图式 2 Lautens 的脱甲基两面针碱合成路线

Scheme 2 Total synthesis of $N$-demethylnitidine by Lautens 


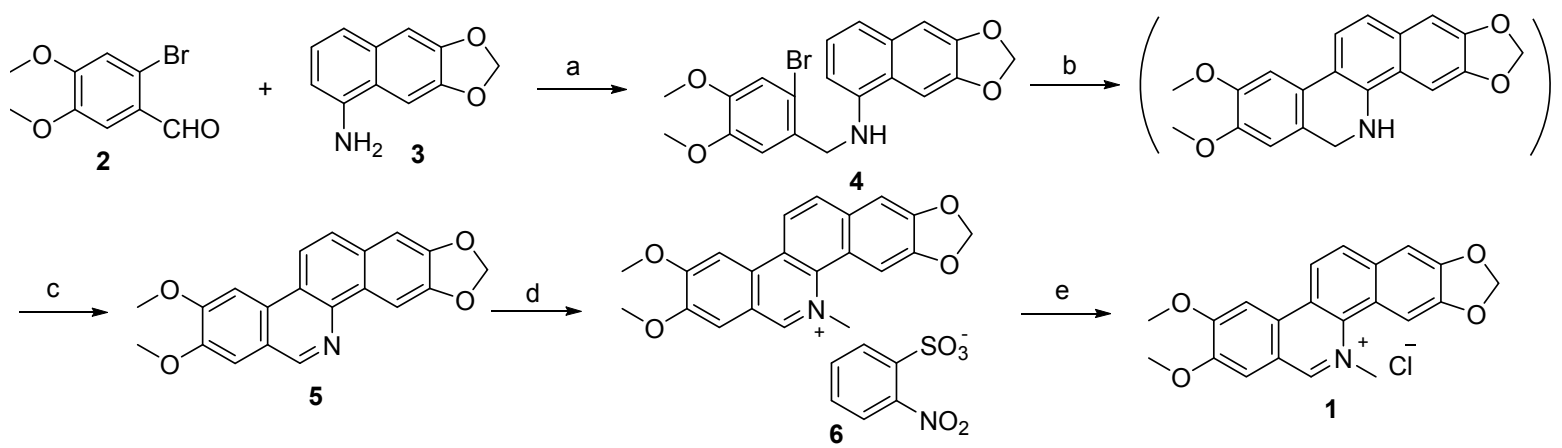

Reagents and conditions: (a) $\mathrm{AcOH}, \mathrm{MeOH}, \mathrm{NaBH}_{3} \mathrm{CN}$, r.t., 17 h, 96\%; (b) $\mathrm{Pd}(\mathrm{OAc})_{2}, \mathrm{P}(\mathrm{o}-\mathrm{tol})_{3}, \mathrm{Ag}_{2} \mathrm{CO}_{3}, \mathrm{DMF}, 4$ h; (c) $\mathrm{MnO}_{2}$; (d) methyl-2-nitrobenzenesulfonate, toluene, r.t., $35 \mathrm{~h}, 98 \%$; (e) $10 \%$ aqueous $\mathrm{HCl}$, r.t., $3 \mathrm{~h}$.

图式 3 本文设计的氯化两面针碱合成路线

Scheme 3 Total synthesis of nitidine chloride in this article

表 1 溶剂、催化剂和配体对反应的影响 ${ }^{a}$

Table 1 Effect of solvent, palladium and ligand on the reaction

\begin{tabular}{lllllcc}
\hline Entry & Palladium & Ligand & Solvent & Temp. & Time/h Yield/\% \\
\hline 1 & $\mathrm{Pd}(\mathrm{OAc})_{2}$ & $\mathrm{PPh}_{3}$ & Bezene & Reflux & 13 & 15 \\
2 & $\mathrm{Pd}(\mathrm{OAc})_{2}$ & $\mathrm{PPh}_{3}$ & Xylene & Reflux & 3 & 20 \\
3 & $\mathrm{Pd}(\mathrm{OAc})_{2}$ & $\mathrm{PPh}_{3}$ & $\mathrm{CH}_{3} \mathrm{CN}$ & Reflux & 12 & 47 \\
4 & $\mathrm{Pd}(\mathrm{OAc})_{2}$ & $\mathrm{PPh}_{3}$ & $\mathrm{DMF}$ & Reflux & 5 & 75 \\
5 & $\mathrm{Pd}_{2}(\mathrm{dba})_{3}$ & $\mathrm{PPh}_{3}$ & $\mathrm{DMF}$ & Reflux & 6 & 65 \\
6 & ${\mathrm{Pd}\left(\mathrm{PPh}_{3}\right)_{4}}_{4}$ & $\mathrm{DMF}$ & Reflux & 4 & 76 \\
7 & $\mathrm{PdBr}_{2}$ & $\mathrm{PPh}_{3}$ & $\mathrm{DMF}$ & Reflux & 7 & 30 \\
8 & $\mathrm{Pd}_{2}(\mathrm{dba})_{3}$ & $\mathrm{PPh}_{3}$ & $\mathrm{DMF}$ & Reflux & 6 & 65 \\
9 & ${\mathrm{Pd}(\mathrm{OAc})_{2}}_{2}$ & $\mathrm{P}(o-\text { tol })_{3}$ & $\mathrm{DMF}$ & Reflux & 4 & 84 \\
10 & $\mathrm{Pd}(\mathrm{OAc})_{2}$ & $\mathrm{BINAP}$ & $\mathrm{DMF}$ & Reflux & 6 & 47 \\
11 & $\mathrm{Pd}(\mathrm{OAc})_{2}$ & $\mathrm{Dppf}$ & $\mathrm{DMF}$ & Reflux & 5 & 51 \\
\hline${ }^{a}$ Reaction conditions: & $\mathrm{Pd}(20 \mathrm{~mol} \%)$, ligand (40 mol\%), $\mathrm{Ag}_{2} \mathrm{CO}_{3}(2$ equiv.).
\end{tabular}

4 8 可以看出, 醋酸钯对分子内的 Heck 偶联反应效果 最好; 篎选几种常用的有机膦配体, 发现三(邻甲苯基) 膦 $\left[\mathrm{P}(o \text {-tol })_{3}\right]$ 对反应的效果明显好于三苯基膦 $\left(\mathrm{PPh}_{3}\right)$ 、联 荎二苯膦(BINAP)和 1,1'-双(二苯基膦)二茂铁(dppf); 反 应时间对反应的影响不很明显. 所以得出 $\mathrm{Pd}(\mathrm{OAc})_{2}$, $\mathrm{P}(o-\text { tol })_{3}$ 和 $\mathrm{DMF}$ 是最好的反应体系, 将产率从最低的 $15 \%$ 提高到 $84 \%$. 关键中间体 4 经过分子内的 Heck 偶联 反应环化, 将二氧化锰氧化直接加入 Heck 偶联反应体 系中, 氧化芳构化得到两面针碱 5 . 两面针碱 5 经过邻 硝基苯磺酸甲酯(methyl $o$-nitrobenzenesulfonate)甲基化 ${ }^{[11]}$, 用氢氧化钠中和产物中的酸性残余物 ${ }^{[12]}$, 后用稀盐 酸酸化得到终产物氯化两面针碱.

\section{2 结论}

本文以 6-溴-3,4-二甲氧基苯甲醛和 6,7-亚甲二氧 基-1-萗胺为起始原料, 应用分子内的 Heck 偶联反应完 成关键的环化反应, 五步反应的总收率为 $55.5 \%$. 在 Suzuki 的合成路线基础上，探索出新的合成方法，而且 操作简单, 反应条件温和, 为合成苯并 $[c]$ 菲啶骨架的化 合物提供了新方法.

\section{3 实验部分}

\section{1 仪器与试剂}

${ }^{1} \mathrm{H}$ NMR 和 ${ }^{13} \mathrm{C}$ NMR 分别由 Bruker-500 型核磁共 振仪在 500 和 $125 \mathrm{MHz}$ 测定, 三甲基硅烷为内标. 质谱 以 Agilent $1100 \mathrm{LC} / \mathrm{MSD}(70 \mathrm{eV})$ 型质谱仪测定. 熔点由 显微熔点测定仪测定. 薄层层析(TLC)和柱层析分别使 用山东青岛海洋化工厂生产的薄层层析硅胶 GF254 型 硅胶和 $200 \sim 300$ 目柱层析硅胶. 实验中所用原料均为 分析纯.

\section{2 实验方法}

3.2.1 N-(2-㴡-4,5-二甲氧基苄基)-苯并 $[2,3-d]-1,3-$ 二 恶茂-5-胺(4)的合成

6-澳-3,4-二甲氧基苯甲醛(2) $2.43 \mathrm{~g}(10 \mathrm{mmol})$ 和

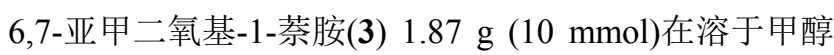
$(20 \mathrm{~mL})$ 中, 用乙酸调节到 $\mathrm{pH} 6$, 在室温搅拌 $30 \mathrm{~min}$. 然 后将 $\mathrm{NaBH}_{3} \mathrm{CN} 1.24 \mathrm{~g}(20 \mathrm{mmol})$ 加入到甲醇中, 在室温 下搅拌 $16 \mathrm{~h}$. 浓缩后经硅胶柱层析[洗脱剂: $V$ (二氯甲 烷) $: V$ (甲醇) $=10:$ 1]纯化得到化合物 $43.73 \mathrm{~g}$, 产率 90\%. ${ }^{1} \mathrm{H}$ NMR (500 MHz, DMSO- $d_{6}$ ) $\delta: 7.65(\mathrm{~s}, 1 \mathrm{H}), 7.14$ (d, $J=5.0 \mathrm{~Hz}, 2 \mathrm{H}), 7.03 \sim 6.91(\mathrm{~m}, 3 \mathrm{H}), 6.52(\mathrm{t}, J=5.6$ $\mathrm{Hz}, 1 \mathrm{H}), 6.18(\mathrm{~d}, J=7.6 \mathrm{~Hz}, 1 \mathrm{H}), 6.06(\mathrm{~d}, J=16.9 \mathrm{~Hz}$, 2H), 4.34 (d, $J=5.6 \mathrm{~Hz}, 2 \mathrm{H}), 3.74(\mathrm{~d}, J=5.2 \mathrm{~Hz}, 3 \mathrm{H}), 3.53$ (s, $3 \mathrm{H}) ;{ }^{13} \mathrm{C}$ NMR (125 MHz, DMSO- $d_{6}$ ) $\delta: 148.8,147.3$, $146.8,143.5,131.4,130.6,125.4,119.7,119.2,119.1$, $116.2,116.0,113.1,112.9,112.8,109.0,104.5,103.7$, 101.4, 99.0, 56.3, 56.0, 47.2; ESI-MS $m / z: 416.1([\mathrm{M}+$ $\left.\mathrm{H}]^{+}\right), 448.1\left([\mathrm{M}+\mathrm{Na}]^{+}\right)$.

\subsection{2 $\mathrm{N}$-脱甲基两面针碱(5)的合成}

在氩气下，将化合物 4 (41.5 $\mathrm{mg}, 0.1 \mathrm{mmol})$, $\mathrm{Pd}(\mathrm{OAc})_{2}(4.44 \mathrm{mg}, 0.02 \mathrm{mmol}), \mathrm{P}(o-\mathrm{tol})_{3}(12.2 \mathrm{mg}, 0.04$ $\mathrm{mmol}$ )和 $\mathrm{Ag}_{2} \mathrm{CO}_{3}(55.2 \mathrm{mg}, 0.2 \mathrm{mmol}$ ) 溶于 $\mathrm{DMF}(5 \mathrm{~mL})$ 
中，加热回流，用薄层色谱监控反应，反应完全后，反 应液冷却到室温, 加入 $\mathrm{MnO}_{2}(48 \mathrm{mg}, 0.55 \mathrm{mmol})$, 继续 搅拌 $1 \mathrm{~h}$, 氧化完全后, 反应液经过硅藻土过滤, 浓缩后 硅胶柱层析[洗脱剂: $V$ (二氯甲烷 $): V($ 甲醇 $)=10 ： 1$ ] 纯 化得到化合物 5, $27.9 \mathrm{mg}$, 产率 84\%. ${ }^{1} \mathrm{H}$ NMR (500 MHz, DMSO- $\left.d_{6}\right) \delta$ : $9.29(\mathrm{~s}, 1 \mathrm{H}), 8.62(\mathrm{~d}, J=9.0 \mathrm{~Hz}, 1 \mathrm{H})$, $8.54(\mathrm{~s}, 1 \mathrm{H}), 8.15$ (s, 1H), $7.94(\mathrm{~d}, J=9.0 \mathrm{~Hz}, 1 \mathrm{H}), 7.69$ (s, 1H), 7.50 (s, 1H), 6.19 (s, 2H), 4.07 (s, 3H), 3.96 (s, 3H); ${ }^{13} \mathrm{C}$ NMR (125 MHz, DMSO- $d_{6}$ ) $\delta$ : 153.5, 149.3, 148.4, $146.3,140.7,140.4,129.7,128.7,126.7,122.3,119.9$, 108.1, 104.9, 103.4, 101.3, 101.5, 56.5, 56.3; ESI-MS $m / z$ : $334.1\left([\mathrm{M}+\mathrm{H}]^{+}\right)$.

\subsection{3 两面针碱邻硝基苯磺酸盐 $(6)$ 的合成}

将化合物 $527.9 \mathrm{mg}(0.083 \mathrm{mmol})$ 和邻硝基苯磺酸 甲酯 $36 \mathrm{mg}(0.166 \mathrm{mmol})$ 溶于甲苯, 回流 $35 \mathrm{~h}$, 产生黄色 沉淀, 冷去至室温, 过滤, 甲苯洗涤, 等到粗产物化合 物 $645.1 \mathrm{mg}$, 产率 98\%. ${ }^{1} \mathrm{H}$ NMR (500 MHz, DMSO- $d_{6}$ ) $\delta: 9.83(\mathrm{~s}, 1 \mathrm{H}), 8.90(\mathrm{~d}, J=9.1 \mathrm{~Hz}, 1 \mathrm{H}), 8.37(\mathrm{~s}, 1 \mathrm{H}), 8.30$ (d, $J=5.9 \mathrm{~Hz}, 1 \mathrm{H}), 7.88(\mathrm{~s}, 1 \mathrm{H}), 7.83 \sim 7.81(\mathrm{~m}, 1 \mathrm{H}), 7.77$ (s, $1 \mathrm{H}), 7.57 \sim 7.52(\mathrm{~m}, 3 \mathrm{H}), 7.50 \sim 7.48(\mathrm{~m}, 1 \mathrm{H}), 6.33(\mathrm{~s}$, $2 \mathrm{H}), 4.88(\mathrm{~s}, 3 \mathrm{H}), 4.21$ (s, 3H), 4.03 (s, 3H).

\section{2 .4 氯化两面针碱(1)的合成}

将粗产物化合物 6 投到 $10 \%$ 盐酸溶液 $12 \mathrm{~mL}$, 室温 搅拌 $3 \mathrm{~h}$, 过滤, 得目标化合物 $\mathbf{1} 26.6 \mathrm{mg}$, 产率 $85 \%$. ${ }^{1} \mathrm{H}$ NMR (500 MHz, MeOD) $\delta$ : 9.63 (s, 1H), 8.75 (d, $J=8.9$ $\mathrm{Hz}, 1 \mathrm{H}), 8.29$ (s, 1H), $8.22(\mathrm{~d}, J=11.1 \mathrm{~Hz}, 2 \mathrm{H}), 7.81(\mathrm{~s}$, 1H), 7.59 (s, 1H), 6.27 (s, 2H), 4.94 (s, 3H), $4.26(\mathrm{~s}, 3 \mathrm{H})$, $4.11(\mathrm{~s}, 3 \mathrm{H}) ;{ }^{13} \mathrm{C}$ NMR (125 MHz, MeOD) $\delta: 170.7,152.3$, $150.1,149.0,133.1,130.2,120.0,118.4,108.0,105.6$, 103.4, 102.9, 102.6, 56.3, 55.6, 50.6; $\quad$ ESI-MS $m / z: 348.1$ $\left([\mathrm{M}-\mathrm{Cl}]^{+}\right)$. HRMS calcd for $\mathrm{C}_{21} \mathrm{H}_{18} \mathrm{NO}_{4} 348.1236$, found 348.1237 .

文献报道的核磁数据 ${ }^{[5]}:{ }^{1} \mathrm{H}$ NMR (DMSO- $\left.d_{6}\right) \delta$ : 9.874 (s, 1H), 8.391(d, $J=8.9 \mathrm{~Hz}, 1 \mathrm{H}), 8.327$ (t, 2H),
7.908 (d, $J=11.1 \mathrm{~Hz}, 2 \mathrm{H}), 7.795$ (s, 1H), 6.355 (s, 2H), 4.902(s, 3H), 4.237 (s, 3H), 4.050 (s, 3H).

辅助材料(supporting information) 各化合物的原始谱 图. 这些材料可以免费从本刊网站 (http://sioc-journal. $\mathrm{cn} /$ )上下载.

\section{References}

[1] Poeta, M. D.; Chen, S. F.; Hoff, D. C.; Dykstra, C. C.; Wani, M. C.; Manikumar, G.; Heitman, J.; Wall, M. E.; Perpect, J. R. Agents Chemother. 1999, 43, 2862.

[2] Hu, J.; Zhang, W. D.; Liu, R. H.; Zhang, C.; Shen, Y. H.; Li, H. L.; Liang, M.J.; Xu, X. K.Chem. Biodiversity 2006, 3, 990.

[3] Gatto, B.; Sanders, M. M.; Yu, C.; Wu, H. Y.; Makhey, D.; LaVoie, E. J.; Liu, L. F. Cancer Res. 1996, 56, 2795.

[4] (a) Gillespie, J. P.; Amoros, L. G.; Stermitz, F. R.; Maestri, G. J. Org. Chem. 1974, 39, 3239.

(b) Nakanishi, T.; Suzuki, M.; Mashiba, A.; Ishikawa, K.; Yokotsuka, T. J. Org. Chem. 1998, 63, 4235.

(c) Enomoto, T.; Girard, A. L.; Yasui, Y.; Takemoto, Y. J. Org. Chem. 2009, 74, 9158.

(d) Larraufie, M.-H.; Derat, E.; Ollivier, F. L.; Lacôte, E.; Malacria, M. Org. Lett. 2010, 12, 5692.

(e) Nakamura, H.; Saito, H.; Nanjo, M. Tetrahedron Lett. 2008, 49, 2697.

[5] Cushman, M.; Cheng, L. J. Org. Chem. 1978, 43, 286.

[6] Nakanishi, T.; Suzuki, M. Org. Lett. 1999, 1, 985.

[7] Nakanishi, T.; Suzuki, M.; Mashiba, A.; Ishikawa, K.; Yokotsuka, T. J. Org. Chem. 1998, 63, 4235.

[8] Blanchot, M.; Candito, D. A.; Larnaud, F.; Lautens, M. Org. Lett. 2011, 13, 1486.

[9] Majumdar, K. C.; Chakravorty, S.; De, N. Tetrahedron Lett. 2008, 49, 3419.

[10] (a)Xu, Z.-H.; Pan, S.; Huang, Y.-G. Chin. J. Org. Chem. 2014, 34, 1391 (in Chinese).

(徐之涵, 潘菼, 黄焰根, 有机化学, 2014, 34, 1391.)

(b) Deng, C.-G.; Li, S.-L.; Liu, X.-W.; Du, H.-G. Chin. J. Org. Chem. 2013, 33, 1741 (in Chinese).

(邓聪迩, 李顺来, 刘祥伟, 杜洪光, 有机化学, 2013, 33, 1741.)

[11] Ramani, P.; Fontana, G. Tetrahedron Lett. 2008, 49, 5262.

[12] Simanek, V.; Preininger, V. Heterocycles 1977, 4, 475. 